\title{
A Novel Approach for Medical Images Noise Reduction Based RBF Neural Network Filter
}

\author{
Mohammed Debakla ${ }^{*}$, Khalifa Djemal'2, Mohamed Benyettou ${ }^{1}$ \\ ${ }^{1}$ MOSIM Laboratory, University of science and Technology Oran, Algeria. \\ 2 IBISC Laboratory, University of Évry Val d'Essonne, France. \\ * Corresponding author. Tel.: +213776446279; email: mohamed.debakla@univ-usto.dz \\ Manuscript submitted January 28, 2015; accepted March 25, 2015. \\ doi: $10.17706 /$ jcp.10.2.68-80
}

\begin{abstract}
This paper is dedicated to the presentation of a Radial basis function neural network (RBFNN) based denoising method for medical images. In the proposed approach, a RBFNN filter is designed where the output of the network is a single denoised pixel and the inputs are its neighborhood in the degraded image. The back-propagation algorithm is used to train the RBFNN filter by minimizing an appropriate error function obtained from the total variation model. The parameters to be adjusted are the weights and the neurons centers of the RBFNN. The considered filter was used to reduce noise from X-ray, MRI and Mammographic medical images giving good results of noise removal when compared to other approaches and using different noise standard deviations.
\end{abstract}

Key words: Noise reduction, medical images, total variation, radial basic fonction neural network, pixel neighborhood.

\section{Introduction}

The quality of digital medical images becomes an important issue. This is due of the widespread of digital imaging. To achieve the best possible diagnosis, medical images should be clear, and without noise and artifacts. While the technologies for acquiring digital medical images continue to improve, resulting in images of higher and higher resolution and quality, removing noise in these images remains one of the major challenges in the treatment of medical imaging, because noise could mask and blur important features in the images. The objective of image denoising is to obtaining the clean image from the noisy one with some knowledge of the degradation process.

There are many existing methods and algorithms for image denoising |1]-[4]. Recently, various types of neural networks have been evaluated for their denoising effectiveness. Radial basis function (RBF) neural networks with their approximation capabilities [5] are very emergent powerful tools. They have the specificity of learning by themselves to extract hidden information in a mass of data, and provide powerful models to the knowledge of a given problem. They are used for classification, prediction and aggregation of data and have been successfully introduced in image denoising [6], [7]. To obtain desired performances from the RBF neural network, weights and Gaussian parameters such us centers and widths have to be adjusted using an appropriate training algorithm.

This paper, presents a new image denoising method by RBF neural network filter based on Total Variation model (TV) minimization [1]. Indeed, the proposed filter; use the neighborhood information of a considered 
pixel as inputs and a single neuron producing the corresponding pixel in its output. The training of the proposed RBF network is achieved by the back-propagation algorithm for determining the optimal weights and centers. This optimal parameters are obtained by minimization of an appropriate error function. The Gaussian widths of RBF are pre-determined by making some assumptions on their values. In the initial step the centers values are computed by K-means clustering algorithm.

The remainder of this paper is organized as follow:

Section 2 presents some related works in the use of neural networks, especially radial basis function neural networks to reduce noise. Section 3 provides the problem formulation of Total variation minimization and the architecture of the proposed RBFNN neural filter and its training algorithm. Section 4 illustrates the application of our RBFNN filter to reduce noise from X-Ray, MRI and Mammographic images and its comparison with some known denoising methods. This last section closed by a study of input size influence of the neural network.

\section{Related Works}

Artificial neural networks (ANN) has seen an increasing use in noise reduction problems. Zhou et al. [8] are the first who proposed the use of the Hopfield neural network (HNN) in image restoration and showed the instability of the original (HNN). They proposed an algorithm to ensure the stability of the HNN. They also proposed the use of simulated annealing algorithm that allows energy increase with a probability decreasing in time so as to converge to a better solution in stochastic sense. These two algorithms have important computation time due to the energy change which checked step by step. Paik and Katsaggelos [9] proposed a Modified Hopfield neural network (MHNN) model for solving the restoration problem which improves the algorithm proposed by Zhou et al. in [8]. The algorithm based on the MHNN ensures network stability without checking energy change step by step, where two new updating schemes (sequential and parallel one) are introduced. However, the convergence proof for the parallel scheme is based on an almost never satisfied condition. Sun Yi [10], [11], presents a Generalized Updating Rule (GUR) of the MHNN for gray image recovery. The stability properties of the GUR are given. It is shown that the neural threshold set up in this GUR is necessary and sufficient for energy decrease with probability one at each update.

Chen et al. [12] introduced an ANN architecture for reducing the acoustic noise level in magnetic resonance (MR) imaging processes. The proposed ANN consists of two cascaded time-delay ANN. The ANN is used as predictor of a feedback active noise control (ANC) system for reducing acoustic noises. Preliminary results also show that, with the proposed ANC system installed, acoustic MR noises are greatly attenuated while verbal communication during MRI sessions is not affected.

Suzuki et al. [13] proposed an analysis method that makes clear the characteristics of the trained NF (i.e. nonlinear filters based on multilayer neural networks) and developed approximate filters that achieves very similar results but is efficient at computational cost. Zhang and Salari [14] have also attempted to denoise images by applying multilayer perceptrons on wavelet coefficients which can be seen as an attempt to incorporate prior knowledge about images. To detect lung nodules overlapped with ribs or clavicles in chest radiographs, Suzuki et al. [15] developed an image processing technique for suppressing the contrast of ribs and clavicles in chest radiographs by means of a multi-resolution massive training artificial neural network (MTANN). They have used a bone images as the teacher oins for training the MTANN. This method has produced a better rib-suppressed images where the soft-tissue opacities were substantially maintained. Hainc and Kukal [16] indicate that artificial neural network can also be used as a kind of a sophisticated nonlinear filter on local pixel neighbourhood $(3 \times 3)$ since linear systems are not good in their sensitivity to impulse (isolated) noise.

Castro et al. [17] proposed to use a multiscale neural network approach for restoring degraded images. 
The method uses a Multilayer Perceptron (MLP) algorithm, trained using synthetic 8-bit gray level image of artificially degraded co-centered circles, with $256 \times 256$ pixels. A convolutional neural network was used to denoise images by jain et al. [18]. They find that convolutional networks provide good results in comparison with wavelet and Markov random field (MRF) methods. Moreover, they find that a convolutional network offers similar performance in the blind denoising compared to other techniques such as non-blind setting. Tarasia et al. [19] have proposed a parallel approach called image decomposition technique to train FLANN (Functional Link Artificial Neural Network) is used for rectifying the corrupted pixels to restore the image; it takes almost $50 \%$ less time than serial approach. Recently a new technique of neural network and fuzzy logic has been proposed by Singh et al. [20] as a tool for image denoising and enhancement, the proposed technique removes the Additive white Gaussian Noise from the Computed Tomography (CT) images and improved their quality. In debakla et al. [21], authors proposed a new image restoration method based on solving PDE with nonlinear model by multilayer neural network basing on the Total Variation (TV) model. The denoising of an image is done by presenting the image pixel by pixel in the neural network. The developed algorithm improves noise reduction and preserves the original geometric characteristics and contrasts of the image.

RBF neural networks have been widely used in image denoising, we can cite the works of Kaoru et al. [6] who proposed a noise reduction filter that can reduce noise components without destroying important image information. The RBF network is then used to recover a high-quality image from the degraded version, and the regularization parameter is adjusted according to local image characteristics. A novel technique for blind image restoration and resolution enhancement based on radial basis function (RBF) neural network was used by Ping and Lei [22]. The RBF network gives a solution of the regularization problem often seen in function estimation with certain standard smoothness functional used as stabilizers. Li-yun et al. [23] have proposed a semi-blind defocused image deconvolution technique based on RBF neural network and iterative Wiener filtering. The RBF neural network is trained in wavelet domain to estimate defocus parameter and after obtaining the point spread function (PSF) parameter, iterative Wiener filter is adopted to complete the restoration. Recently Shamik et al. [7] proposed a proficient method for uniform motion blur parameter estimation for blind restoration of motion blurred barcode images. By considering the fact that barcode images have high linear singularities, ridgelet transform has been used.

Most of the studded methods that we have cited have advantages and inconvenient. Certain of them provide satisfactory filtering results but some details are lack such as important object contours. In this aim, and to preserve contours we propose to use a new denoising formulation based on TV model minimization where the resolution is obtained using RBF neural network. Indeed, this method of noise reduction also starts with a noisy image but without any a priori information on the original image.

\section{Radial Basic Function Neural Network Filter (RBFNNF)}

We present a description of the RBFNNF technique in this section. We start from the formulation of the image denoising problem by minimizing the TV model under constraints. Secondly, we propose the structure and training of RBFNNF used to minimizing an appropriate error function obtained from the total variation model in order to reduce noise.

\subsection{Problem Formulation}

Total variation (TV) regularization has been extremely successful in a wide variety of denoising problems. It has been introduced for image denoising and reconstruction in a known paper of Rudin et al. [1] with the minimization of the following functional: 


$$
F(u)=\int_{\Omega}|D u|+\lambda\left\|u_{0}-u\right\|^{2} d x d y
$$

where $\int_{\Omega}|D u|$ represents the TV model of the image $u$. If the image $u$ is regular, the equation (1) becomes only $\int_{\Omega}|\nabla u| d x$. Rudin et al. [1] considered that the noise which corrupted the image is distinguished from noiseless one by the size of total variation, which is defined as $\int_{\Omega} \sqrt{u_{x}^{2}+u_{y}^{2}} d x d y$, where $\Omega$ denotes the image domain $u_{x}$ and $u_{y}$ denote the corresponding partial differentiation. Consequently, they propose to restore a noisy and blurred image by minimizing total variation given by:

$$
\min _{u} \int_{\Omega} \sqrt{u_{x}^{2}+u_{y}^{2}} d x d y
$$

Under constraints:

$$
\left\{\begin{array}{l}
\int_{\Omega} \frac{1}{2}\left(u(x, y)-u_{0}(x, y)\right)^{2} d x d y=\sigma^{2} \\
\int_{\Omega}\left(u(x, y)-u_{0}(x, y)\right) d x d y=0
\end{array}\right.
$$

where $u_{0}(x, y)$ represents the given observed image, which is considered to be corrupted by a Gaussian noise of variance $\sigma^{2}$ and $u(x, y)$ denote the desired clean image.

To minimize (2) Rudin et al. [1] have applied the Euler-Lagrange equation under the two constraints in (3), they obtain the following equation:

$$
\frac{\partial}{\partial x}\left(\frac{u_{x}}{\sqrt{u_{x}^{2}+u_{y}^{2}}}\right)+\frac{\partial}{\partial y}\left(\frac{u_{y}}{\sqrt{u_{y}^{2}+u_{y}^{2}}}\right)-\lambda\left(u-u_{0}\right)=0
$$

where $\lambda$ the Lagrange multiplier is given by:

$$
\lambda=\frac{1}{2 \sigma^{2}} \int\left[\sqrt{u_{x}^{2}+u_{y}^{2}}-\left(\frac{\left(u_{0}\right)_{x} u_{x}}{\sqrt{u_{x}^{2}+u_{y}^{2}}}\right)+\left(\frac{\left(u_{0}\right)_{y} u_{y}}{\sqrt{u_{x}^{2}+u_{y}^{2}}}\right)\right]
$$

An image denoising problem can be transformed to an optimization problem. In our assumption, and from (4), we can formulate the image denoising problem as minimizing the following error function:

$$
E(x, y)=\frac{\partial}{\partial x}\left(\frac{u_{x}}{\sqrt{u_{x}^{2}+u_{y}^{2}}}\right)+\frac{\partial}{\partial y}\left(\frac{u_{y}}{\sqrt{u_{y}^{2}+u_{y}^{2}}}\right)-\lambda\left(u-u_{0}\right)
$$

where $u_{x}$ and $u_{y}$ are discretizations of the horizontal and vertical derivatives. A difficulty with TV is that, it has a derivative singularity when $u$ is locally constant. To avoid this, some algorithms regularize TV by introducing an additional small parameter $\varepsilon>0, \sqrt{u_{x}^{2}+u_{y}^{2}+\varepsilon}$ 


\subsection{Structure of RBFNNF}

To minimize (6), we propose to use a Radial Basic Function neural network (RBFNN). Generally, a RBFNN consists of three layers: the input layer, the RBF layer (hidden layer) and the output layer. The inputs of hidden layer are the linear combinations of scalar weights and the input vector $x\left[x_{1}, x_{2}, \ldots, x_{n}\right]^{T}$, where the scalar weights are assigned unity values. Thus the whole input vector appears to each neuron in the hidden layer. The incoming vectors are mapping by the radial basis functions in each hidden node. The output layer yields a vector $y\left[y_{1}, y_{2}, \ldots, y_{m}\right]^{T}$ for m outputs by linear combination of the outputs of the hidden nodes to produce the final output. Fig. 1 presents the structure of a single output RBFNN; the network output can be obtained by:

$$
y=f(x)=\sum_{i=1}^{h} w_{i} \phi_{i}(x)
$$

where $f(x)$ is the final output, $\phi($.$) denotes the radial basis function of the i^{\text {th }}$ hidden node, $w_{i}$ denotes the hidden-to-output weight corresponding to the $i^{- \text {th }}$ hidden node, and $\mathrm{h}$ is the total number of hidden nodes.

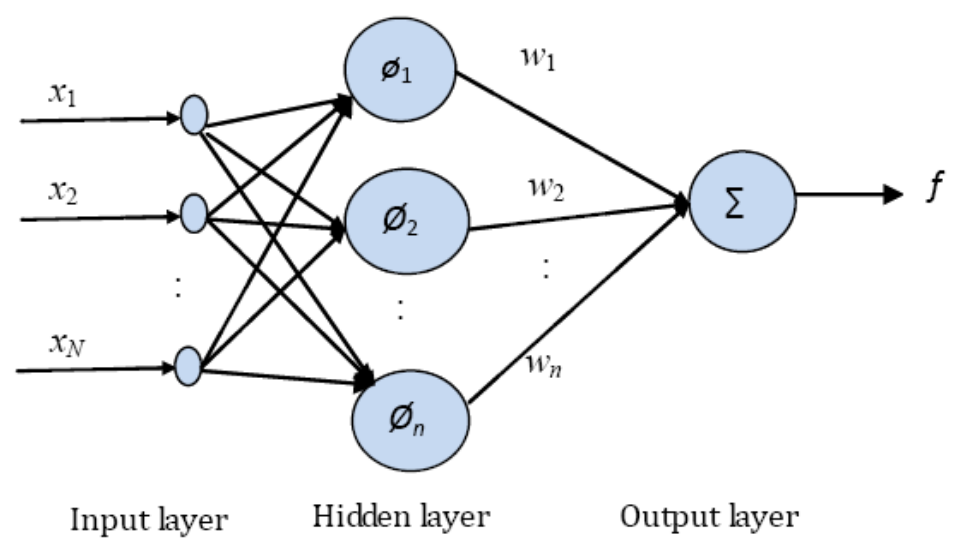

Fig. 1. Structure of a single output RBFNN.

A radial basis function is a multidimensional function that describes the distance between a given input vector and a pre-defined center vector. There are different types of radial basis function. A normalized Gaussian function usually is used as the radial basis function, it is given by:

$$
\phi_{i}(x)=\exp \left[\frac{-\left\|x-c_{i}\right\|}{2 \sigma_{i}^{2}}\right]
$$

where $c_{i}$ and $\sigma_{i}$ denote the center and spread width of the $\mathrm{i}^{\text {th }}$ node, respectively. The parameters of an RBFNN have to be initialized before the network is trained. The weights $\{w j, j=1,2, \ldots, M\}$ can be initialized to either small random values or zeros. The initial centers can be determined, for example, by K-means clustering of a number of the input samples. Spread initial values $\sigma_{i}$ could be chosen as the average of the nearest-neighbor distances among the initialized centers and trained later, or fixed during the training process; this parameter is generally fixed after multiple learning experimentations.

We take the assumption that the output of the RBFNNF is an image corresponding to the desired image $u$. So we provided as inputs to the RBFNNF the degraded image $u_{0}$. The output of the network is formulated by: 


$$
u=N\left(u_{0}, w_{i}, c_{i}\right)
$$

where $u_{0}$ is the noisy image represented by each intensity of pixel $(x, y)$ and his neighbourhood (Fig. 2).

The gray level of the output of each pixel $u_{0}(x, y)$ is calculated by:

$$
N(x, y)=\left(\sum_{i=1}^{h} w_{i} \exp \left[\frac{-\left\|V_{0}(x, y)-c_{i}\right\|}{2 \sigma_{i}^{2}}\right]\right)
$$

where $V_{0}(x, y)$ is the gray level values of the neighbourhood pixels correspond to the pixel $u_{0}(x, y)$. The window size of the neighbourhood could be $3 \times 3,5 \times 5,7 \times 7 \ldots$

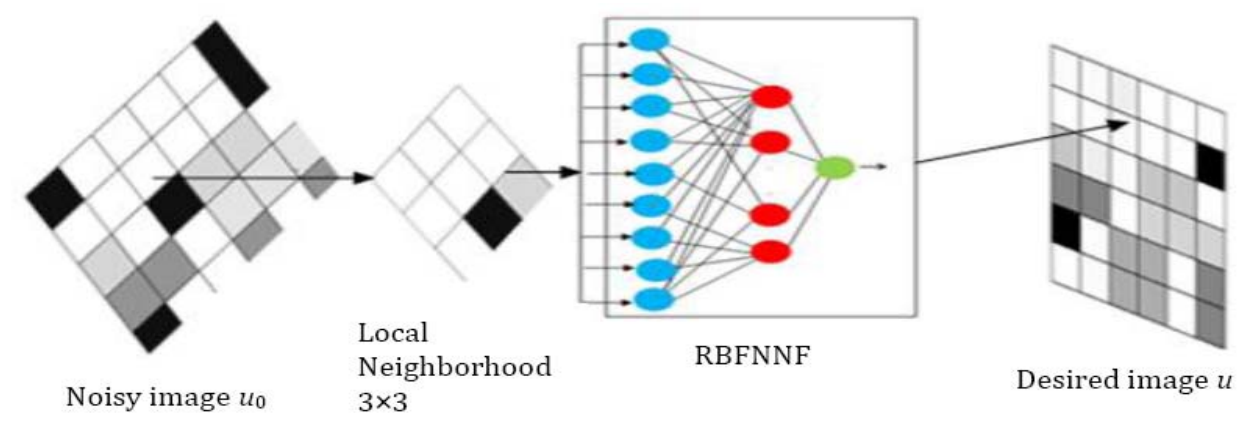

Fig. 2. RBFNN Filter of our approach.

\subsection{Training of RBFNNF}

Once all pixels of the noisy image are presented to the network, an image $u$ is obtained. We assume that this image satisfies the equation (4). This image is replaced in equation (5) to get $\lambda$, then in equation (6) for calculating the error function $E$ which is provided by the network. The RBFNNF is trained by the back-probagation algorithm [24] until the maximum iterations number is reached or the obtained error is less than the convergence error threshold $E_{C}$. This algorithm adjusts the parameters $w_{i}$ and $c_{i}$ according to:

$$
\begin{aligned}
& w_{i}(t+1)=w_{i}(t)-\eta_{1} \Delta w_{i}(t) \\
& c_{i}(t+1)=c_{i}(t)-\eta_{2} \Delta c_{i}(t)
\end{aligned}
$$

where $w_{i}(t+1)$ and $c_{i}(t+1)$ represent the values of parameters (weights and centres) of next iteration and $w_{i}(t)$ and $c_{i}(t)$ are their values for current iteration. $\eta_{1}$ and $\eta_{2}$ are positive learning rates.

The parameters variation $\Delta w_{i}$ and $\Delta c_{i}$ are obtained by minimization of error $E(x, y)$ presented in equation (6) using the following equation:

$$
\begin{aligned}
\Delta w_{i} & =\sum_{x, y} \frac{\partial E(x, y)}{\partial w_{i}} \\
\Delta c_{i} & =\sum_{x, y} \frac{\partial E(x, y)}{\partial c_{i}}
\end{aligned}
$$


To compute $\Delta w_{i}$ and $\Delta c_{i}$ and for the simplicity of the calculation, we denote the two terms of equation (6) as follows:

$$
\begin{aligned}
& \frac{u_{x}}{\sqrt{u_{x}^{2}+u_{y}^{2}}}=f(x, y) \text { and } \frac{u_{y}}{\sqrt{u_{x}^{2}+u_{y}^{2}}}=g(x, y) \\
& \frac{\partial}{\partial x}\left(\frac{u_{x}}{\sqrt{u_{x}^{2}+u_{y}^{2}}}\right)=f_{x}, \frac{\partial}{\partial y}\left(\frac{u_{y}}{\sqrt{u_{x}^{2}+u_{y}^{2}}}\right)=g_{y}
\end{aligned}
$$

Equation (6) becomes:

$$
E(x, y)=f_{x}+g_{y}-\lambda\left(u-u_{0}\right)
$$

From these considerations, the parameters variation terms $\Delta w_{i}$ and $\Delta c_{i}$ can be formulated as follows:

$$
\begin{gathered}
\frac{\partial E(x, y)}{\partial w_{i}}=\frac{\partial f_{x}}{\partial w_{i}}+\frac{\partial g_{y}}{\partial w_{i}}-\frac{\partial \lambda}{\partial w_{i}}\left(u-u_{0}\right)-\lambda \frac{\partial u}{\partial w_{i}} \\
\frac{\partial E(x, y)}{\partial c_{i}}=\frac{\partial f_{x}}{\partial c_{i}}+\frac{\partial g_{y}}{\partial c_{i}}-\frac{\partial \lambda}{\partial c_{i}}\left(u-u_{0}\right)-\lambda \frac{\partial u}{\partial c_{i}}
\end{gathered}
$$

All terms of the equations (16) and (17) are calculated from the value of $\frac{\partial u}{\partial w_{i}}$ and $\frac{\partial u}{\partial c_{i}}$ so it is necessary to provide firstly these derivatives. Under our assumptions and considerations in (9) and as $N(x, y)$ is considered in (10), under these conditions, the derivatives can be given as follows:

$$
\frac{\partial u}{\partial w_{i}}=\frac{\partial N}{\partial w_{i}}, \frac{\partial u}{\partial c_{i}}=\frac{\partial N}{\partial c_{i}}
$$

So, the derivative of the desired image with respect to the weights is given by:

$$
\frac{\partial u}{\partial w_{i}}=\exp \left(\frac{-\left\|v_{0}(x, y)-c_{i}\right\|}{2}\right)
$$

and, the derivative of the desired image with respect to the centers is given by:

$$
\frac{\partial u}{\partial c_{i}^{j}}=\frac{1}{2} w_{i} \frac{\left(v_{0}^{j}-c_{i}^{j}\right)}{\left\|v_{0}-c_{i}\right\|} \exp \left(\frac{-\left\|v_{0}-c_{i}\right\|}{2}\right)
$$

$\left\{c_{i}^{j}, j=1,2, \ldots, s\right\}$ where $\mathrm{s}$ is the window size of the neighbourhood pixels. As explained above, the optimal width of RBF Gaussian function $\sigma_{i}$ is experimentally fixed to 1 .

From the obtained derivations $\frac{\partial u}{\partial w_{i}}$ and $\frac{\partial u}{\partial c_{i}}$ we can easily calculate $\Delta w_{i}$ and $\Delta c_{i}$.

\section{Experimental Results}

In this section, some experimental results are presented to evaluate the performance of RBFNNF approach. The denoising performance of this approach is compared with other denoising methods using their optimal parameters: minimizing TV model of Rudin et al. [1], Wiener filter, and Multilayer Neural Network (MLP approach) Debakla et al. [21]. To do this, three kinds of medical images, (X-ray, MRI and Mammographic images) are used. The ISNR is used as a quality metric to compare objectively the 
performances of image denoising algorithms; it represents the amount of noise removed from the degraded image. If ISNR increases, then the result of denoising is best. This metric, using the restored image, is given by:

$$
\operatorname{ISNR}(f, u)=10 \log _{10} \frac{\sum_{i, j}\left[f(i, j)-u_{0}(i, j)\right]^{2}}{\sum_{i, j}[f(i, j)-u(i, j)]^{2}}
$$

where $f(i, j), u_{0}(i, j)$ and $u(i, j)$ denote the original, degraded and denoised images, respectively.

The Normalized Mean Square Error (NMSE) as another measure of quality is also used. If the value of NMSE decreases, the denoising is better. NMSE is given by:

$$
\operatorname{NMSE}(f, u)=\frac{\sum_{i, j}[f(i, j)-u(i, j)]^{2}}{\sum_{i, j}[f(i, j)]^{2}}
$$
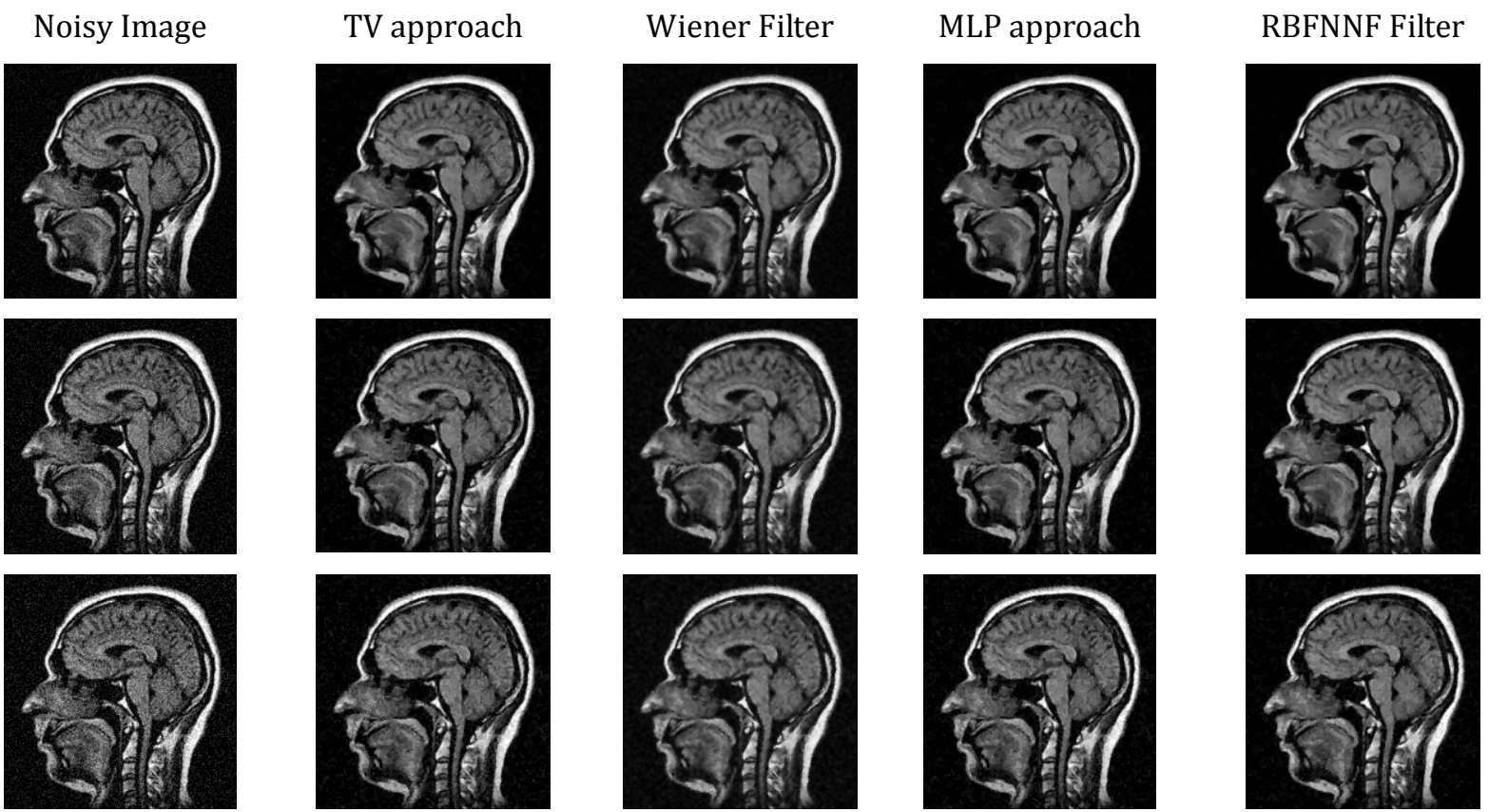

Fig. 3. MRI image denoising: The columns from left to right show the noisy image and the restored images by the total variation, Wiener Filter, MLP neural network and RBFNNF approach. The rows from top to down are showing the experiments with different standard deviations of noise $(\sigma=15,20$, and 25).

The first denoising experiment is shown in Fig. 3 for this experiment, using a MRI image of $220 \times 220$ pixels taken from public link http://irm-bird.com/index.php?path=Public/Exemples. White Gaussian noise with three different standard deviations $\sigma=15,20$ and 25 are added. The columns of Fig. 3 from left to right show the noisy image and the restored images by the total variation with: $n=400, \mathrm{dt}=0.001$, alpha $=250$ and epsilon $=0.1$, Wiener Filter, MLP neural network and RBFNNF approach. The used window is $3 \times 3$ for Weiner Filter and for our RBFNNF approach. The corresponding ISNR and NMSE values are shown in Table 1.

The proposed method (RBFNNF) has given a good visual quality with strong noise removal and also more details are preserved. From Table 1, we can see that the RBFNN filter has obtained the best metric values for the three noise standard deviation $\sigma=15,20$ and 25 . 
Table 1. The ISNR and NMSE Values of White Gaussian Noise Reduction for MRI Image

\begin{tabular}{lllll}
\hline Image & & $\sigma=15$ & $\sigma=20$ & $\sigma=25$ \\
\cline { 2 - 5 } WV) approach & ISNR & 3.5781 & 3.8502 & 3.7723 \\
\cline { 2 - 5 } & NMSE & 0.0117 & 0.0196 & 0.0307 \\
\hline \multirow{2}{*}{ MLP approach } & ISNR & 2.3139 & 3.3652 & 3.8965 \\
\cline { 2 - 5 } & NMSE & 0.0156 & 0.0219 & 0.0298 \\
\hline \multirow{2}{*}{ RBFNNF Filter } & ISNR & 4.6410 & 4.9614 & 4.8812 \\
\cline { 2 - 5 } & NMSE & 0.0115 & 0.0181 & 0.0277 \\
\hline \hline
\end{tabular}

Noisy Image
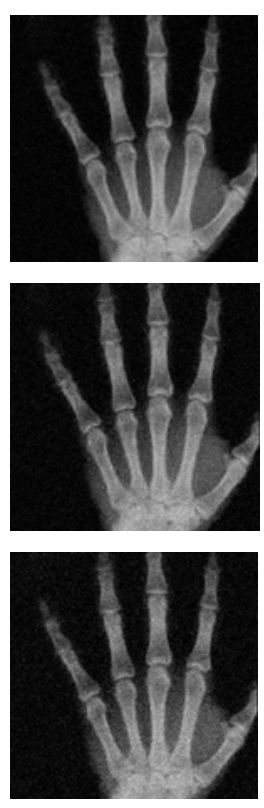

TV approach
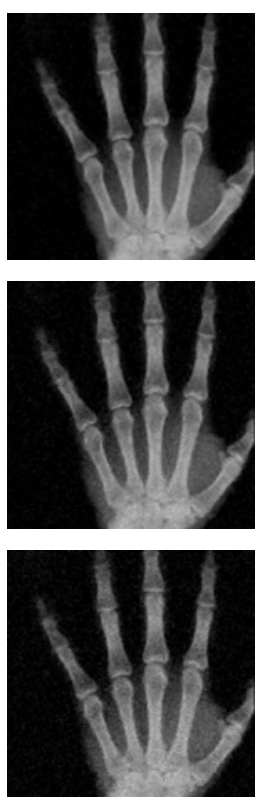
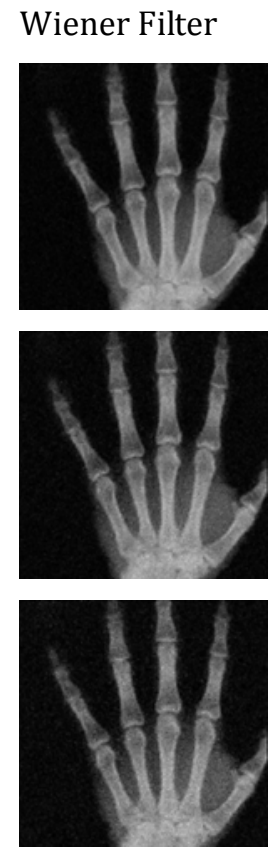

MLP approach
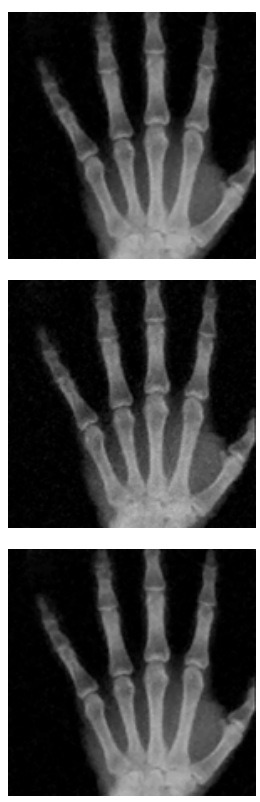

RBFNNF Filter
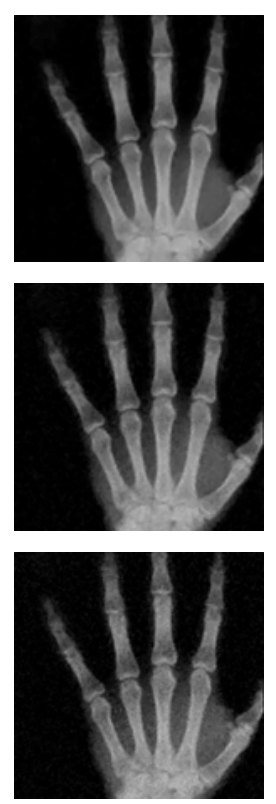

Fig. 4. X-ray image denoising: The columns from left to right show the noisy image and the restored images by the total variation, Wiener Filter, MLP neural network and RBFNNF approach. The rows from top to down are showing the experiments with different standard deviations of noise $(\sigma=15,20$, and 25).

Table 2. The ISNR and NMSE Values of White Gaussian Noise Reduction for X-ray Image

\begin{tabular}{lllll}
\hline \hline Image & & $\sigma=15$ & $\sigma=20$ & $\sigma=25$ \\
\hline \multirow{2}{*}{ TV)approach } & ISNR & 7.9673 & 6.7432 & 5.7707 \\
\cline { 2 - 5 } & NMSE & 0.0060 & 0.0133 & 0.0253 \\
\hline \multirow{2}{*}{ Wiener Filter } & ISNR & 4.9572 & 4.9032 & 4.8218 \\
\cline { 2 - 5 } & NMSE & 0.0107 & 0.0189 & 0.0299 \\
\hline \multirow{2}{*}{ MLP approach } & ISNR & 9.2629 & 7.9364 & 6.8102 \\
\cline { 2 - 5 } & NMSE & 0.0048 & 0.0104 & 0.0203 \\
RBFNNF Filter & ISNR & $\mathbf{1 0 . 7 2 3 6}$ & $\mathbf{9 . 4 0 9 6}$ & $\mathbf{8 . 1 9 2 1}$ \\
\cline { 2 - 5 } & NMSE & $\mathbf{0 . 0 0 2 8}$ & $\mathbf{0 . 0 0 6 7}$ & $\mathbf{0 . 0 1 3 8}$ \\
\hline \hline
\end{tabular}

As second experimentation, our approach is applied to denoising X-ray image of $461 \times 610$ pixels taken from http://lifeinthefastlane.com/resources/normal-x-ray-database, under the same settings used in the first experimentation, the results are given in Fig. 4. 
The presented results show the good performance of our algorithm, especially the preservation of discontinuities. Moreover the geometric characteristics such as corners and edges and originals contrast are well restored. For purposes of comparison, the results of noise reduction by the considered methods are shown in Table 2.

It is clear from result of Table 2 that the proposed approach (RBFNNF) has enhanced the noise reduction due to the ability of the training algorithm when compared to the other filters. Indeed, the values of ISNR metric are the best ones for our algorithm when applied to denoise the three noisy images $(10.7236,9.4096$ and 8.1921 respectively).

Thirdly, we have applied the RBFNNF on mammography image of $258 \times 241$ pixels taken from http://www.mammoimage.org/databases/, the results are given in Fig. 5. It is clear from these figures that the performance of the new method is effective in different images. It can be seen from Table 4 that RBFNNF is better than the others in image quality and the two metrics (ISNR and MNSE) show its efficiency.

Table 3. The ISNR and NMSE Values of White Gaussian Noise Reduction for Mammographic Image

\begin{tabular}{lllll}
\hline \hline Image & & $\sigma=15$ & $\sigma=20$ & $\sigma=25$ \\
\hline \multirow{2}{*}{$($ TV)approach } & ISNR & 5.3307 & 5.3543 & 4.9563 \\
\cline { 2 - 5 } & NMSE & 0.0048 & 0.0086 & 0.0195 \\
\hline \multirow{2}{*}{ Wiener Filter } & ISNR & 5.0524 & 6.1389 & 6.6124 \\
\cline { 2 - 5 } & NMSE & 0.0052 & 0.0072 & 0.0099 \\
\hline \multirow{2}{*}{ MLP approach } & ISNR & 8.2564 & 10.4088 & 12.5264 \\
\cline { 2 - 5 } & NMSE & 0.0034 & 0.0027 & 0.0025 \\
\hline \multirow{2}{*}{ RBFNNF Filter } & ISNR & $\mathbf{8 . 4 2 5 7}$ & $\mathbf{1 0 . 7 1 7 1}$ & $\mathbf{1 3 . 0 6 3 2}$ \\
\cline { 2 - 5 } & NMSE & $\mathbf{0 . 0 0 2 4}$ & $\mathbf{0 . 0 0 2 5}$ & $\mathbf{0 . 0 0 2 2}$ \\
\hline \hline
\end{tabular}

Noisy Image
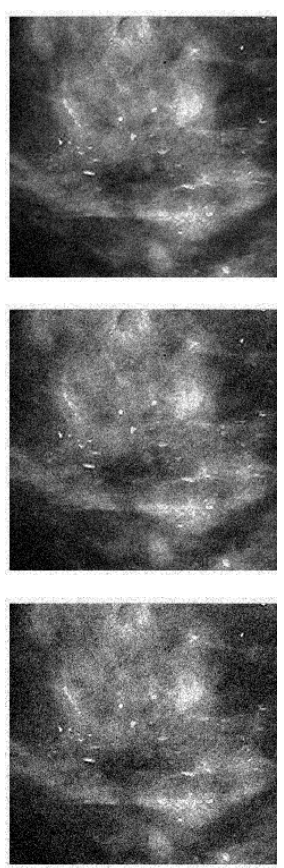

TV approach
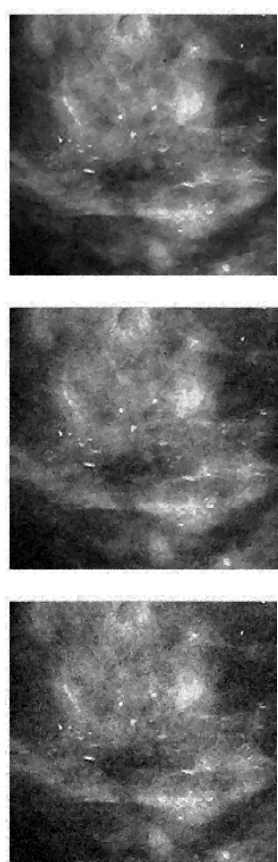

Wiener Filter
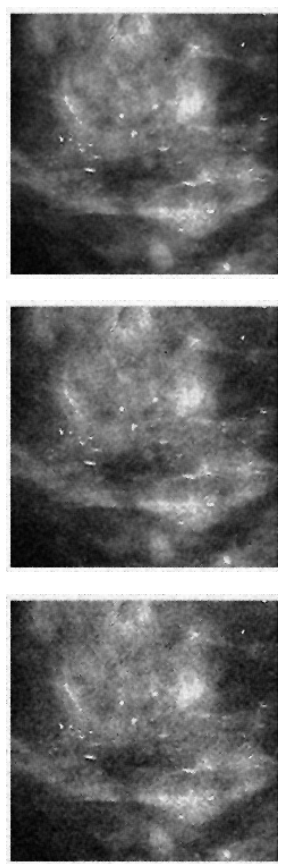

MLP approach
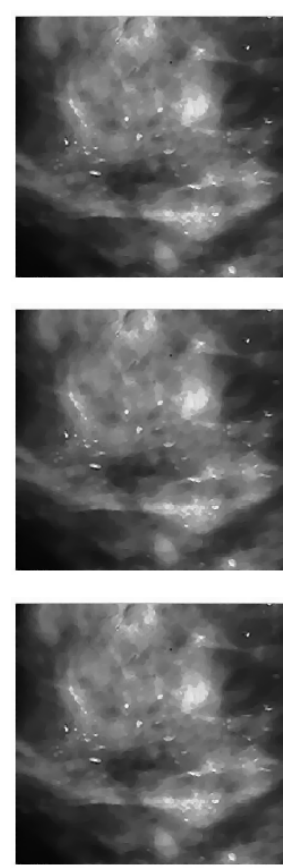

RBFNNF Filter
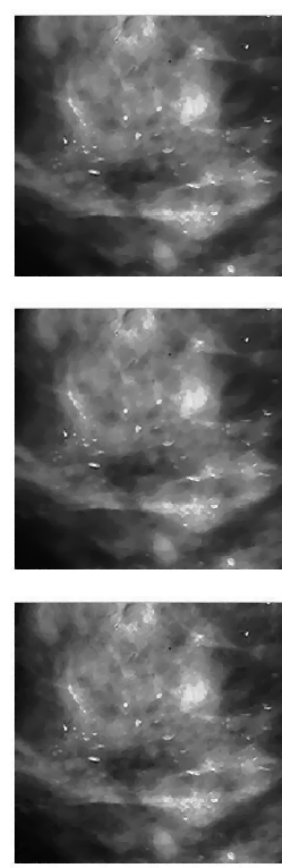

Fig. 5. Mammographic image denoising: The columns from left to right show the noisy image and the restored images by the total variation, Wiener Filter, MLP neural network and RBFNNF approach. The rows from top to down are showing the experiments with different standard deviations of noise $(\sigma=15,20$, and 25).

In the last experimentation, the influence of window size over the RBFNN filter is studied where the 
neighborhood pixels are used as inputs of the RBFNN filter to denoise images; the window size could be $3 \times 3,5 \times 5,7 \times 7 \ldots$

The proposed approach is applied to denoise MRI image with standard deviations $\sigma=15$ using window sizes $3 \times 3$ and $5 \times 5$, it is compared to Weiner filter using the same window sizes.

The resulted denoised images are presented in Fig. 6. These results are summarized in Table 4 using ISNR and NMSE metrics.

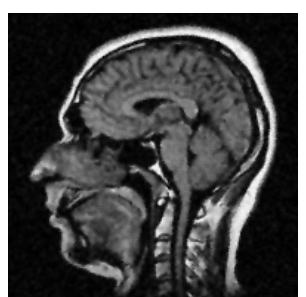

a) Wiener filter $3 \times 3$

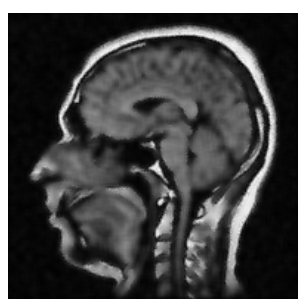

b) Wiener filter $5 \times 5$

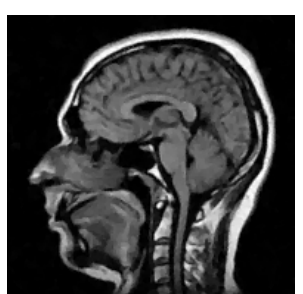

c) RBFNNF $3 \times 3$

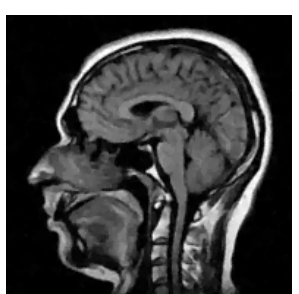

d) RBFNNF $5 \times 5$

Fig. 6. RM image denoising with: a) Wiener filter $3 \times 3$, b) Wiener filter $5 \times 5$, c) RBFNNF $3 \times 3$, d) RBFNNF $5 \times 5$.

Table 4 . The ISNR and NMSE Values of the RBFNNF and Wiener Filter with $3 \times 3$ and $5 \times 5$ Neighborhood Sizes

\begin{tabular}{|c|c|c|c|c|}
\hline & Wiener filter $3 \times 3$ & RBFNNF $3 \times 3$ & Wiener filter $5 \times 5$ & RBFNNF $5 \times 5$ \\
\hline ISNR & 2.3139 & 4.6876 & 0.2562 & 4.7638 \\
\hline MNSE & 0.0156 & 0.0090 & 0.0282 & 0.0089 \\
\hline
\end{tabular}

It could be noticed that, for the Weiner filter, the denoising performance has decreased (Table 4) when we grow up the window size to $5 \times 5$, in the same time, some blurred regions have emerged in the denoised image.

In this experimentation, when using our RBFNN filter, we can see that the size of the window does not significantly affect the quality of denoised image even it lightly increases ( e.g ISNR value was 4.6876 for $3 \times 3$ neighborhood, for $5 \times 5$ it becomes 4.7638 ).

\section{Conclusion}

In this work, a denoising approach for medical images has been presented. The proposed approach uses a new filtering formulation based on TV model minimization where the resolution is obtained using RBF neural network. The training of the RBFNN filter is carried out by back-propagation algorithm to adjust the nodes centers and the hidden layer weights.

The proposed filter was used to reduce noise from X-ray, MRI and Mammographic medical images giving good visual quality with strong noise removal and also more details are preserved.

The influence of the window size over the denoising quality of our approach is also studied, where we have deduced that the RBFNN filter is less sensitive to the change of the window size relative to wiener filter.

Comparison of our approach with some known denoising methods has shown that the performance of the RBFNNF is promising.

\section{References}

[1] Rudin, L., Osher, S., \& Fatemi, E. (1992). Nonlinear total variation based noise removal algorithms. Physica., 60, 259-268.

[2] Katsaggelos, A. K., Biemond, J., Schafer, R. W., \& Mersereau, R. M. (1999). A regularized iterative image 
restoration algorithm. IEEE Trans. Signal Processing, 39, 914-929.

[3] Huiyan, W., \& Jia, Z. (Mar. 2013). Comparative study of tongue image denoising methods. Journal of Computers, 8(3), 787-794.

[4] Sun, Z. G., Chen, S. C., \& Qiao, L. S. (Nov. 2014). A two-step regularization framework for non-local means. Journal of Computer Science and Technology, 29(6).

[5] Haykin, S. (1999). Neural Networks: A Comprehensive Foundation (2nd ed.). N.J. Prentice Hall.

[6] Kaoru, I., Youji, I., \& Hajime, M. (2003). Image restoration using the RBF network with variable regularization parameters. Neurocomputing, 50, 177-191.

[7] Shamik, T., Vidhya, P. S., Biradar, S. R., \& Ajay, S. (2014). Blind Restoration of motion blurred barcode images using ridgelet transform and radial basis function neural network. Electronic Letters on Computer Vision and Image Analysis, 13(3), 63-80.

[8] Zhou, Y. T., Vaid, A., \& Jenkins, B. K. (1988). Image restoration using a neural network. IEEE Trans. Acoust Speech Signal Processing, 36, 114.

[9] Paik, J. K., \& Katsaggelos, A. (1992). Image restoration using a modified hopfield network. IEEE Transactions on Image Processing, 1(1), 49-63.

[10] Sun, Y. A. (1998). Generalized updating rule for modified hopfield neural network for quadratic optimization. Neurocomput., 19, 133-143.

[11] Sun, Y. (2000). Hopfield neural network based algorithms for image restoration and reconstruction Part II: Algorithms and simulation. IEEE Trans. Signal Processing, 48, 2105-2118.

[12] Chen, G. K., Chiueh, T. D., \& Chen, J. H. (1999). Active cancellation system of acoustic noise in MR imaging. IEEE Transactions on Biomedical Engineering, 46(2), 186-191.

[13] Suzuki, K. H., \& Sugie, N. I. (2002). Efficient approximation of neural filters for removing quantum noise from images. IEEE Trans. Signal Process, 50(7), 1787-1799.

[14] Zhang, S., \& Salari, E. (2005). Image denoising using a neural network based non-linear filter in wavelet domain. Proceedings of IEEE International Conference on Acoustics, Speech, and Signal Processing: Vol. 2 (p. 989).

[15] Suzuki, K., Abe, H., MacMahon, H., \& Doi, K. (2006). Image processing technique for suppressing ribs in chest radiographs by means of massive training artificial neural network (MTANN). IEEE Transactions on Medical Imaging, 25(4).

[16] Hainic, L., Kukal, J., \& Role, J. (2006). Of robust processing in ANN de-noising of 2D image. Neural Network World, 16(2), 163-76.

[17] Castro, A. P. A., Drummond, I. N., \& Silva, J. D. S. (2008). A multiscale neural network method for image restoration. TEMA Tend. Mat. Apl. Comput., 9(1), 41-50.

[18] Jain, V., \& Seung, H. (2008). Natural image denoising with convolutional networks, advances. Neural Information Processing Systems, 21, 769-776.

[19] Tarasia, N., Mishra, M. K., Dash, P. C., \& Samal, S. K. (2011). A parallel approach to train flann for an adaptive filter. Proceedings of the 3rd International Conference on Advanced Computer Control.

[20] Singh, R., Sapra P., \& Verman, V. (2013). Advanced technique of de-noising medical images using ANFIS. International Journal of Science and Modern Engineering, 1(9).

[21] Debakla, M., Djemal, K., \& Benyettou, M. (2014). Image restoration using multilayer neural networks with minimization of total variation approach. IJCSI International Journal of Computer Science Issues, 11(2).

[22] Ping, G., \& Lei, X., (2004). Blind image restoration based on rbf neural networks', image processing: algorithms and systems III. Proceedings of SPIE: Vol. 5298.

[23] Su, L.-Y., Li, F.-L., Xu, F., \& Liu, Y.-R. (2008). Defocused image restoration using RBF network and 
iterative wiener filter in wavelet domain. Proceedings of International Congress on Image and Signal Processing (pp. 311-315).

[24] Rumelhart, D. E., Hinton, G. E., \& Williams, R. J., (1986). Learning internal representations by error propagation. Parallel Distributed Processing, 1, 318-362.

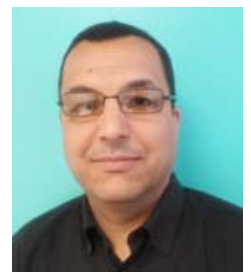

Mohammed Debakla is a PhD student in computer science at University of Science and Technology of Oran (USTO), Algeria. He received the diploma of engineering in computer science from the University of Oran (USTO), Algeria in 1996. He received the diploma of teaching in computer science from the University of Oran (USTO), Algeria, in 2005. He is a teacher at the University of Mascara, Algeria since 2005. His research interests are in the field of image processing and optimization methods.

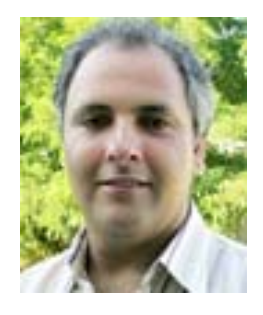

Khalifa Djemal received his diploma degree in optical, image and signal processing in 1999 from the National School of Physics at the University of Marseille, France and his Ph.D. degree in image and signal processing in 2002, from the University of Toulon, France. Since 2003, he is an associate professor at the Electrical Engineering Department of the Institute of Technology at the University of Evry Val d'Essonne, France. He works now within the S.I.M.O.B team of the IBISC Laboratory. His current research interests are in the areas of image and data processing (restoration, segmentation, clusteringand CBIR). Dr. Djemal chaired the International Conference on Image Processing Theory, Tools and Applications IPTA, in 2008, 2010 and 2012, and also International Workshop on Medical Image Analysis and Description for Diagnosis Systems, MIAD, in 2009, 2010 and 2011. He was the chair of some special sessions in a number of conferences. He acted as the technical chairman for a number of conferences. He is a reviewer for a number of international journals and conferences.

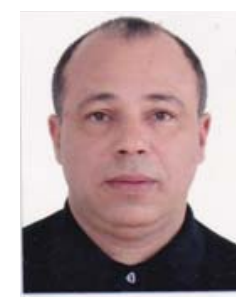

Mohamed Benyettou is a professor in the Department of Computer Science, University of Sciences and Technologies Mohamed Boudiaf USTO, ORAN, Algeria. He's also a director of laboratory of modeling and optimization LAMOSI. His main research interests are artificial intelligence, image processing, optimization methods and so on. 